\title{
Provision of boundary conditions for a convection-permitting ensemble: comparison of two different approaches
}

\author{
C. Marsigli, A. Montani, and T. Paccagnella \\ ARPA-SIMC, HydroMeteoClimate Service of ARPA Emilia-Romagna, Bologna, Italy \\ Correspondence to: C. Marsigli (cmarsigli@arpa.emr.it) \\ Received: 26 July 2013 - Revised: 20 December 2013 - Accepted: 9 January 2014 - Published: 12 March 2014
}

\begin{abstract}
The current resolution of the operational global models favours the possibility of driving convectionpermitting limited-area model (LAM) simulations directly, sparing the necessity for an intermediate step with a coarserresolution LAM. Though the resolution of global ensemble systems is generally lower than that of deterministic ones, it is also possible to consider this opportunity in the field of ensemble forecasting. The aim of this paper is to investigate the effect of this choice for driving a convection-permitting ensemble based on the COSMO model, for a specific application, namely the forecast of intense autumn precipitation events over Italy. The impact of the direct nesting in the ECMWF global ensemble is compared to a two-step nesting, which makes use of a LAM ensemble system with parametrised convection. Results show that the variability introduced in the geopotential field by the direct nesting is usually contained within the uncertainty described by the standard ensemble, and differences between pairs of members following different nesting approaches are generally smaller than the ensemble error, computed with respect to analysis. The relation between spread and error is even improved by the direct nesting approach. In terms of precipitation, it is found that the forecasts issued by members with different nesting approaches generally have differences at spatial scales between 16 and $180 \mathrm{~km}$, depending on the case, hence not negligible. Nevertheless, the skill of the LAM ensemble precipitation forecasts, evaluated by means of an objective verification, is comparable. Therefore, the overall quality of the $2.8 \mathrm{~km}$ ensemble for the specific application is not deteriorated by the provision of lower resolution lateral boundary conditions directly from the global ensemble.
\end{abstract}

\section{Introduction}

The current resolution of operational global circulation models (GCM) paves the way towards driving convectionpermitting limited-area model (LAM) integrations directly, with lateral boundary conditions (LBCs) provided by a global model. Presently, high-resolution LAM runs are generally nested in coarser-resolution integrations of the same model. The intermediate step with a coarser-resolution run is usually performed to ensure a ratio of spatial resolutions between nested models in the range of $2: 1-5: 1$. The aim is to reduce the loss of information due to nesting which would affect the nested model in the case of a larger resolution gap (see for example Warner et al., 1997 and Denis et al., 2001).

With the resolution increase of the GCMs, the intermediate step with a coarser-resolution LAM could be avoided, with some potential benefits. First of all, removing one step in the nesting procedure would decrease the time needed to perform the whole cascade up to the convection-permitting integration, with a consequent reduction of the computational costs. This would be most beneficial in an operational environment, requiring timely delivery of products. Secondly, one shortcoming of the intermediate LAM run is that nowadays this usually runs in the so-called "grey zone", where convection is partly resolved and partly sub-grid. This is recognised to be a delicate issue for many models (see e.g. Gerard et al., 2009). On the other hand, the coarser spatial and temporal resolution of the LBCs provided by a global model could introduce errors in the forecast, especially if the LAM domain is small, as is often the case for convection-permitting models. Some studies have addressed this issue.

As for the spatial resolution of LBCs, de Elia et al. (2002) showed that the quality of the LAM simulation is rather insensitive to the resolution of the driving data. However, this 
is true if boundaries are set far from the region of interest, which is usually not true for convection-permitting simulations. Temporal resolution is also an issue, since LBCs are often produced at much lower frequency than the time step used in the LAM. If information changes rapidly at the boundary, then the LBCs might not reflect the actual changes in the state of the atmosphere (Termonia et al., 2009). Termonia (2003) found that the use of a coupling update interval of $3 \mathrm{~h}$ has a detrimental effect on the forecast quality of a very intense storm, affecting significantly the depth of the lowpressure system. Davies (2014) showed that, with an $11 \mathrm{~km}$ resolution model, 3-hourly LBCs already lead to a loss of information with respect to hourly LBCs at a $12 \mathrm{~h}$ forecast range. Amengual et al. (2007) found that for regional climate model (RCM) runs the update frequency of LBCs has a larger impact on the downscaling results than their spatial resolution. Nevertheless, the ratio between the two factors is dependent on the actual resolutions involved and on the forecast range. As for the relation between the two effects and their impact on LAM ensembles, Nutter et al. (2004) showed that the impact of coarsely resolved LBCs or temporal interpolation of LBCs on error growth is quite similar. Both effects act to remove small-scale features from the external fields passing through the lateral boundary, thereby constraining ensemble dispersion. The impact of coarsely resolved LBCs has been identified as stronger than the one of temporal interpolation when the LBC update frequency is reasonably high.

In this work, the issue of $\mathrm{LBC}$ resolution is considered in the framework of ensemble forecasting. The aim of this paper is to investigate the effect of the provision of LBCs to a LAM ensemble run at a convection-permitting resolution by a global ensemble, compared with providing LBCs from an intermediate LAM ensemble run at coarser resolution. The analysis is confined to a specific meteorological situation, namely autumn precipitation cases over the Mediterranean.

Aiming at the development of an ensemble system at the convection-permitting scale over Italy, ARPA-SIMC has implemented an experimental ensemble based on the COSMO model (Steppeler et al., 2003) during SOP (Special Observation Period) 1.1 of the Hymex Project (HYMEX, 20102020). The ensemble, named COSMO-H2-EPS (COSMO Hymex $2.8 \mathrm{~km}$ Ensemble Prediction System, Marsigli et al., 2013), consists of 10 runs of the COSMO model at $2.8 \mathrm{~km}$ horizontal resolution, with 50 levels in the vertical, and receives perturbed ICs and LBCs from the first 10 members of COSMO-LEPS (COSMO Limited-area Ensemble Prediction System, Montani et al., 2011). COSMO-LEPS, running at $7 \mathrm{~km}$ horizontal resolution with parametrised convection, in turn receives initial and boundary conditions from the global ensemble of ECMWF, referred to as ENS. ENS is currently running with an approximate horizontal resolution of $32 \mathrm{~km}$, which is planned to increase to about $20 \mathrm{~km}$ in 2015 . This may enable direct use of LBCs from ENS members to drive ensemble systems for the convection-permitting scale (2-3 km).
In this work, it is studied how the performance of the COSMO-H2-EPS ensemble varies if the $2.8 \mathrm{~km}$ runs receive ICs and BCs from the ENS members directly, skipping the intermediate step with COSMO-LEPS at $7 \mathrm{~km}$. The aim is to evaluate the effect of removing this step, heading for the future operational set-up of the ensemble, which will benefit from the resolution increase of ENS, up to $20 \mathrm{~km}$. Though in the present configuration the resolution gap between driving model $\left(32 \mathrm{~km}\right.$, retrieved on a grid of $0.25^{\circ}$ mesh size $)$ and driven model $(2.8 \mathrm{~km})$ is quite high, it is believed that an assessment of the difference between the two nesting procedures on the specific ensemble application can provide scientific guidance useful for defining the operational set-up. It is also worth mentioning that, in its operational configuration, the $2.8 \mathrm{~km}$ ensemble will not use as ICs the downscaled ENS analyses, since high-resolution perturbed initial conditions will be provided by a LETKF scheme developed in the COSMO consortium (KENDA system, Reich et al., 2011), which is currently under testing.

The paper is organised as follows: in the next section, the configuration of the ensembles is described. Then, in Sect. 3 results are commented on, divided into an analysis of fields of geopotential at $500 \mathrm{hPa}$ and precipitation. Finally, conclusions are drawn in Sect. 4.

\section{Configuration of the ensemble systems}

COSMO-H2-EPS is an ensemble system specifically designed for the Hymex Project, which was run regularly during SOP 1.1, taking place from 5 September to 6 November 2012. The ensemble consists of 10 runs of the COSMO model at $2.8 \mathrm{~km}$ horizontal resolution, with 50 levels in the vertical, over a domain including most of the target areas of the project. In particular, it covers a large part of Italy, Switzerland and the French Mediterranean coast (Fig. 1). The model domain contains $399 \times 412$ grid points. The ensemble was run once per day, starting at 12:00 UTC, for a forecast range of $36 \mathrm{~h}$.

Initial and boundary conditions are provided by the first 10 members of COSMO-LEPS. COSMO-LEPS receives, in turn, ICs and BCs from 16 members of the ECMWF ENS, selected as the most representative members of the full ensemble through an ensemble reduction algorithm (Molteni et al., 2001; Marsigli et al., 2001). Therefore, perturbed ICs of COSMO-H2-EPS are also derived from ECMWF ENS ones by means of a two-step interpolation: first from the ENS archiving grid to the COSMO-LEPS grid at $7 \mathrm{~km}$ and then from it to the COSMO-H2-EPS grid at $2.8 \mathrm{~km}$. This implies that no data assimilation is performed for the two ensembles; initial conditions are simply downscaled from global analyses. Model perturbations are also applied to the $2.8 \mathrm{~km}$ runs, since few parameters of the COSMO physics schemes (turbulence, microphysics, soil scheme) are set to values different from the default ones in the 10 COSMO runs. It should 
be highlighted that this same model perturbation technique is adopted in COSMO-LEPS, with some differences in the parameters which are perturbed. These pertubations affect the model run starting from the first time step, but do not affect the analysis, therefore ICs are not changed due to model perturbation. For a comprehensive description of the two systems the reader is referred to Marsigli et al. (2013) for COSMO-H2-EPS and to Montani et al. (2011) for COSMOLEPS.

A second experimental ensemble, named EXP-H2-EPS, has been implemented for the present study. It has been run for 21 cases of the SOP 1.1 period, selected as events of moderate to intense (observed) precipitation. A description of the meteorological situation during the SOP and of a few severe events can be found in Ducrocq et al. (2013) and Ferretti et al. (2013) (the latter focussing on Italy).

The ensemble configuration of EXP-H2-EPS is identical to that of COSMO-H2-EPS in terms of resolution, domain, model set-up, and parameter perturbation. The only difference is in the way ICs and BCs are provided: for EXP-H2-EPS they are derived directly from 10 members of ENS, the same members that have been selected to drive the first 10 COSMO-LEPS operational runs. Therefore, pairs of members of EXP-H2-EPS and COSMO-H2-EPS can be compared directly: the $i$ th member of the former ensemble is driven by an ENS member, while the $i$ th member of the latter ensemble is driven by the $i$ th COSMO-LEPS member, which was in turn driven by this same ENS member. It should be underlined that the temporal resolution of the LBCs is also different: hourly BCs are provided to COSMO-H2-EPS by COSMO-LEPS, while 3-hourly BCs are provided to EXPH2-EPS by ENS.

\section{Comparison of the two ensembles}

\subsection{Analysis of $500 \mathrm{hPa}$ geopotential}

A quantitative assessment of the differences between the geopotential fields at $500 \mathrm{hPa}$ predicted by the $2.8 \mathrm{~km}$ ensemble runs, when driven by COSMO-LEPS members and when driven by ENS members, is provided here.

First, the spread and the root-mean-square error (RMSE) of the ensemble mean in terms of geopotential at $500 \mathrm{hPa}$ are computed for the 21 cases and over the whole domain. Ensemble spread has been computed as the root-mean-square (RMS) distance of all the ensemble members from the ensemble mean. RMSE of the ensemble mean has been computed against the operational ECMWF analyses interpolated on the $2.8 \mathrm{~km}$ grid. Since analyses are available only every $6 \mathrm{~h}$, RMSE has also been computed every $6 \mathrm{~h}$, while spread has been computed hourly. The relation between spread and error is usually regarded as an indicator of the capability of the ensemble to represent the forecast error through the dispersion of the members. The spread matching the error guar-

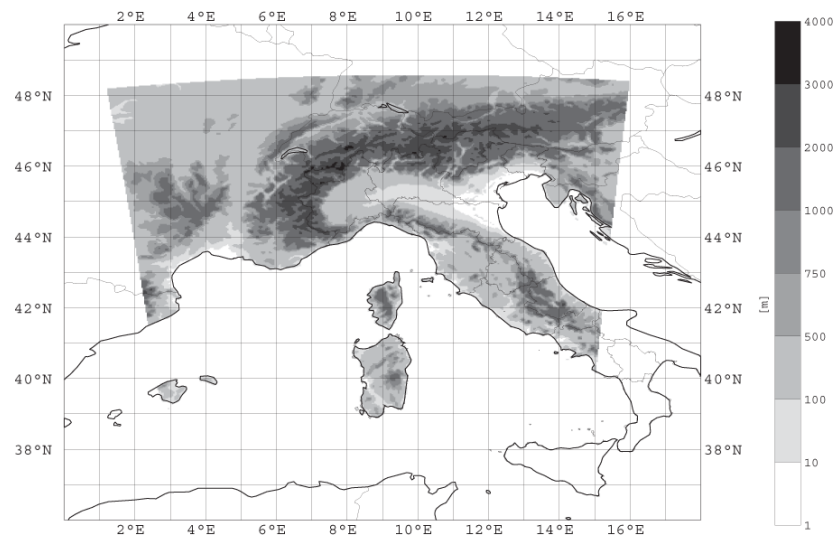

Fig. 1. Orography of the COSMO model at $2.8 \mathrm{~km}$ horizontal resolution, also showing the extension of the integration domain of COSMO-H2-EPS.

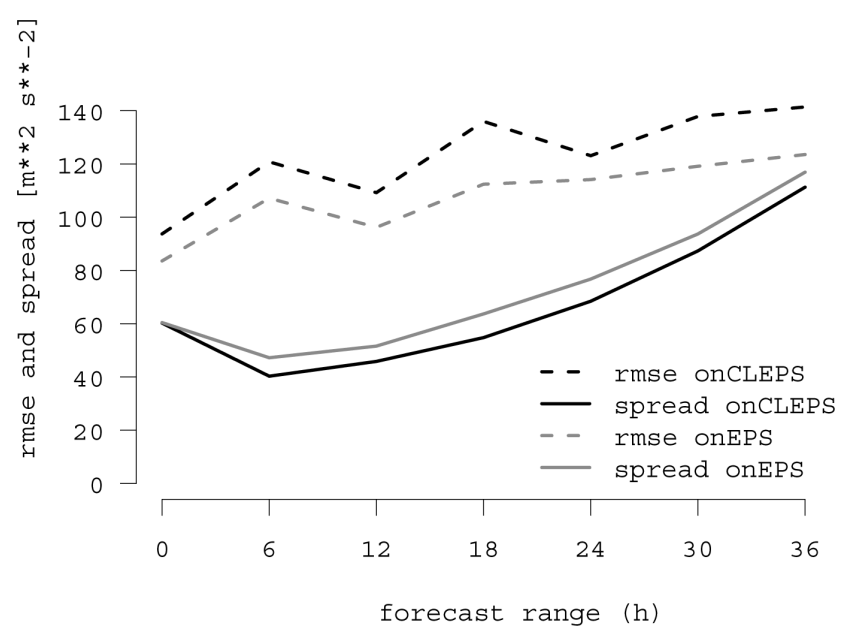

Fig. 2. RMSE of the ensemble mean (dashed lines) and ensemble spread (solid lines) for COSMO-H2-EPS (black, labelled "onCLEPS") and EXP-H2-EPS (grey, labelled "onEPS"), in terms of geopotential at $500 \mathrm{hPa}$.

antees that the ensemble variability allows one to find, on average, the true atmospheric state among the states predicted by the ensemble (e.g. Buizza et al., 2005).

The spread and error of the two systems are compared in Fig. 2. EXP-H2-EPS has slightly more spread than COSMOH2-EPS, with a similar increase with the forecast range. The coincidence of the two spread values at initial time is due to the fact that the initial conditions of the two ensembles are the same ECMWF ENS perturbed analyses, downscaled at $2.8 \mathrm{~km}$ with an interpolation performed either in one step (for EXP-H2-EPS) or in two steps (for COSMO-H2-EPS). The relatively large value may be ascribed to some noise due to the interpolation itself (visible in the z500 maps, not shown).

The RMSEs of the ensemble mean of the two systems increase with lead time, with lower error values for EXP-H2EPS with respect to COSMO-H2-EPS. Therefore, the direct 
nesting allows one to improve the spread-error relation in terms of geopotential at $500 \mathrm{hPa}$, evaluated over the full period and over the whole domain.

In order to check whether the direct nesting causes problems in particular meteorological situations, error and spread have also been computed case by case. First, the RMS difference between the geopotential field forecasted by each member of the COSMO-H2-EPS ensemble and by the correspondent member of the EXP-H2-EPS ensemble is computed. This is performed for each case separately, for each forecast hour (from 0 to $36 \mathrm{~h}$ ) and over the whole integration domain. RMS differences are shown in Fig. 3 as thin solid lines, while the thick dotted line is the average RMS difference, computed between all pairs of members. Results are shown for 4 cases only, selected as representative of the whole sample.

In order to provide a reference against which to evaluate the magnitude of these differences, the spread of the two ensembles has also been computed for each case. The spread is regarded as a measure of the uncertainty expressed by each ensemble. Therefore a difference between pairs of members smaller than the spread would not be detected in the standard ensemble configuration. The spread is plotted as thick solid lines, black for COSMO-H2-EPS and grey for EXPH2-EPS. Finally, the RMSE of the ensemble mean is also computed for each case, and it is shown in Fig. 3 as a dashed line, black for COSMO-H2-EPS and grey for EXP-H2-EPS. RMSE provides the ultimate limit for the RMS differences between pairs of members, since it quantifies the error of the ensemble: until the difference induced by the different nesting approach is smaller than the error of the ensemble, it is not actually possible to establish if this difference is detrimental to the forecast.

In all cases, the spread of each ensemble generally lies among the larger RMS differences between pairs of members and is larger than the average RMS difference. This indicates that the variability introduced by nesting the $2.8 \mathrm{~km}$ model on ENS directly is usually included within the uncertainty described by the reference ensemble (COSMO-H2-EPS). Furthermore, the RMS differences between pairs of members are generally smaller than the RMS error of the ensemble mean, with the few exceptions discussed below. This indicates that in most cases the impact of the different nesting is lower than the forecast error of the reference system.

Nevertheless, some differences can also be identified. The behaviour of the major part of cases ( 15 cases) is close to the one shown in Fig. 3a, relative to the 2012092512 case. The spread is smaller than the error of the ensemble mean for both ensembles, the two spread curves remaining quite close together for the entire forecast range. The differences between pairs of members are generally small, and do not give rise to additional uncertainty with respect to that represented by the COSMO-H2-EPS ensemble. In a few cases among these 15, the RMS differences can increase up to the level of the RMSE for a few hours during the forecast range.

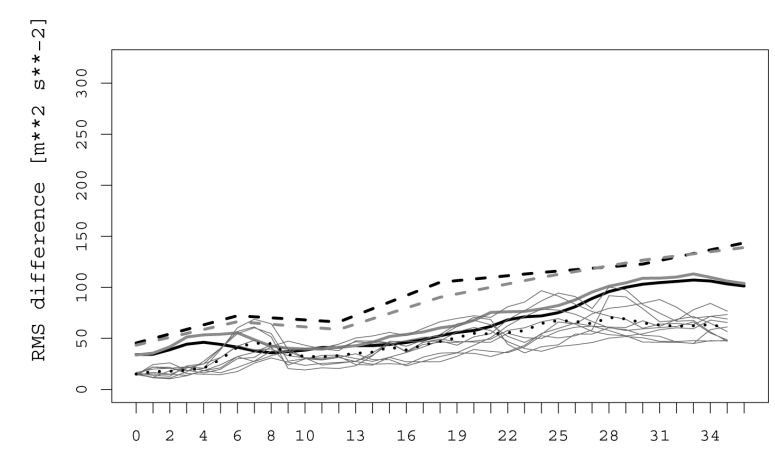

(a)

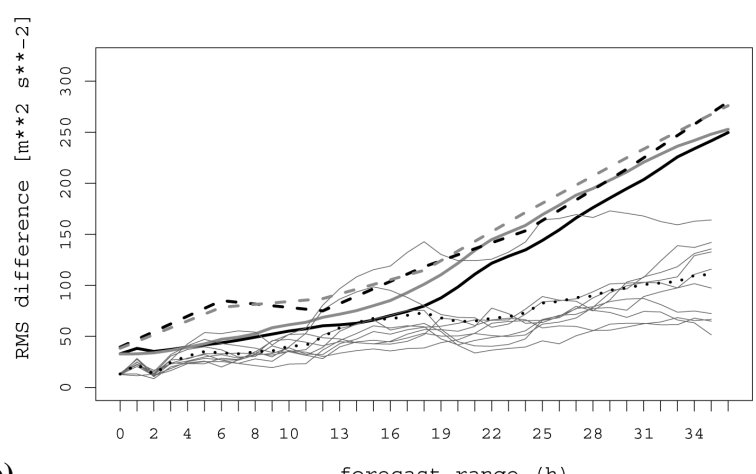

(b)

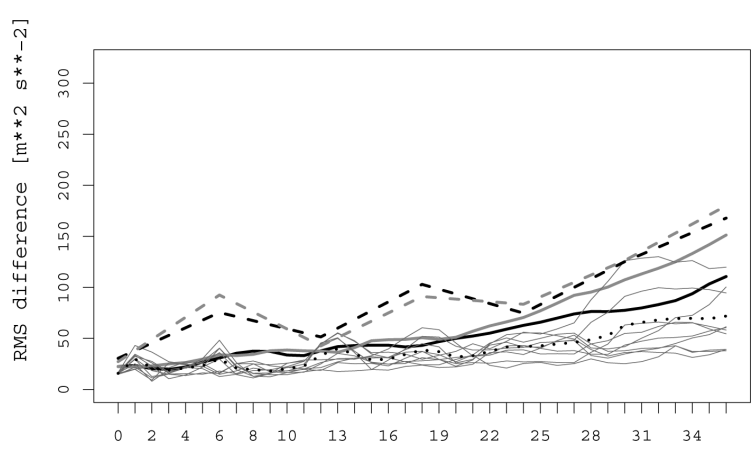

(c)

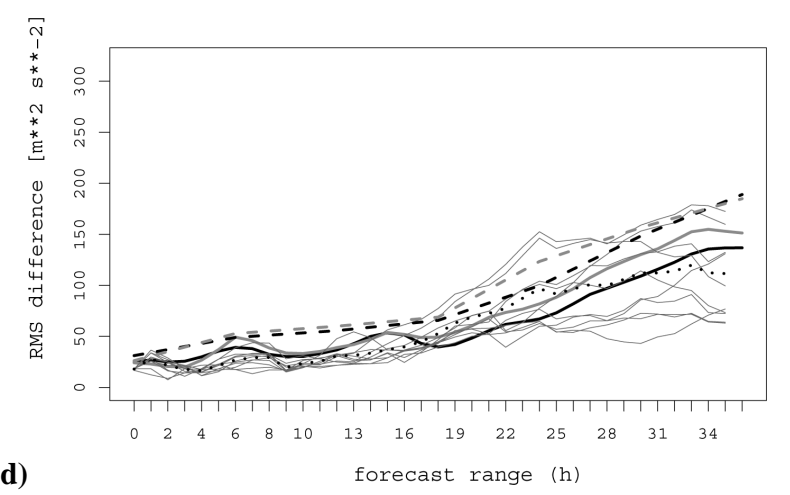

Fig. 3. RMS differences between pairs of correspondent members (thin solid), average RMS difference between all pairs (thick dotted), RMS spread (thick solid) and RMSE of the ensemble mean (thick dashed), as a function of the forecast range. RMS spread and error lines are black for COSMO-H2-EPS and grey for EXPH2-EPS. Plots are relative to four cases only: 2012092512 (a), 2012101412 (b), 2012102512 (c), and 2012103012 (d). 
In the 2012101412 case (Fig. 3b), the spread increases quite sharply after $18-21 \mathrm{~h}$ of simulation, gaining distance from the RMS differences, which become negligible, with the exception of one pair of members. The spread curves roughly match the error curves. In this case, a trough enters the domain from the northwest corner after about $18 \mathrm{~h}$ of integration. The z500 field forecasted by member 1 of EXPH2-EPS (not shown) reproduces the observed trough and its evolution quite well, at difference with the corresponding member of COSMO-H2-EPS. The other pairs of members do not differ that much, but provide less skillful predictions. In the 2012102512 case (Fig. 3c), the RMS differences between members are generally smaller than the spread and quite below the RMSE values. Also in this case, a marked spread increase is observed for EXP-H2-EPS after $20 \mathrm{~h}$, leading to a better match to the RMSE. The meteorological situation is similar to the previous one, with one member of EXP-H2-EPS performing better than the others and better than the corresponding COSMO-H2-EPS member. In both the 2012101412 and 2012102512 cases, additional spread is gained with the direct nesting approach, with almost no increase in the RMSE, mainly due to the better performance of one EXP-H2-EPS member. A similar feature has been detected in two other cases (not shown).

The 2012103012 case (Fig. 3d) offers the most notable exception to the behaviour identified so far. The spread increases with the forecast range, especially after $18 \mathrm{~h}$, in agreement with the increase in the forecast error. Nevertheless, some members have differences larger than the ensemble spread, so that the variability introduced by the direct nesting can be detected as an "outlier" with respect to the variability described by the COSMO-H2-EPS ensemble. Furthermore, two pairs of members have differences larger than the RMSE. Since the RMSE of EXP-H2-EPS is higher that the RMSE of COSMO-H2-EPS, it is concluded that for this case additional error is introduced by the direct nesting. In this case, a deep low-pressure system over the western Mediterranean develops and enters the domain approximately at the $18 \mathrm{~h}$ forecast range. Three members of EXP$\mathrm{H} 2$-EPS provide a poor description of the position and intensity of the low, unlike the correspondent COSMO-H2-EPS members. For the other ensemble members, differences are smaller. Similar behaviour is found on the following day, for the 2012103112 case (not shown).

The same analysis has also been performed in terms of mean sea level pressure (Fig. 4), in order to assess the robustness of the previous analysis.

Results are generally in good agreement with those obtained in terms of geopotential at $500 \mathrm{hPa}$. In the 2012092512 case (Fig. 4a), few RMS differences reach values close to or even above the RMSE. For most of the cases similar to this, though, RMS differences are well below these values. In the 2012103012 case (Fig. 4d), RMS differences of a few pairs of members are also quite high in terms of MSLP, confirming

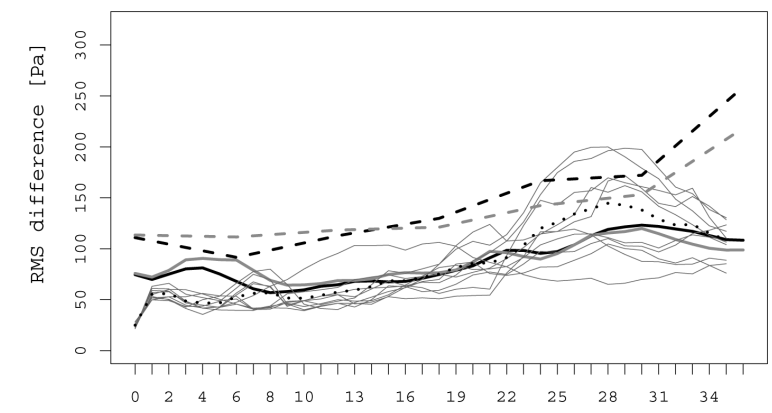

(a)

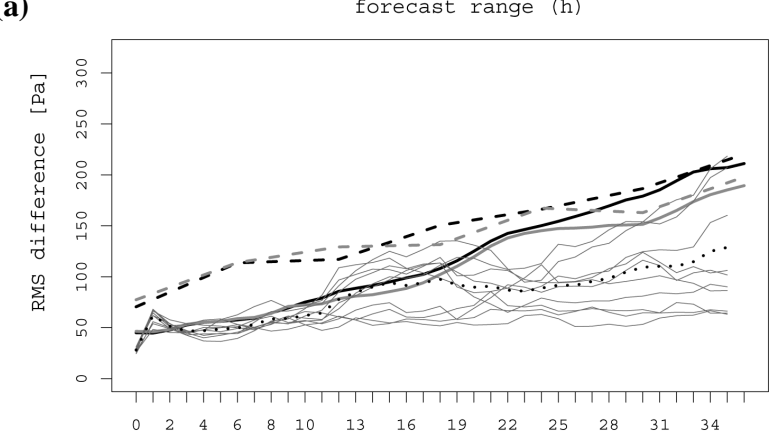

(b)

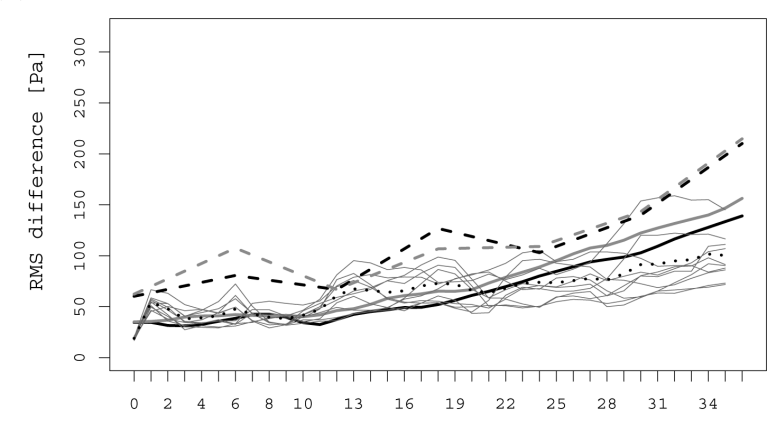

(c)

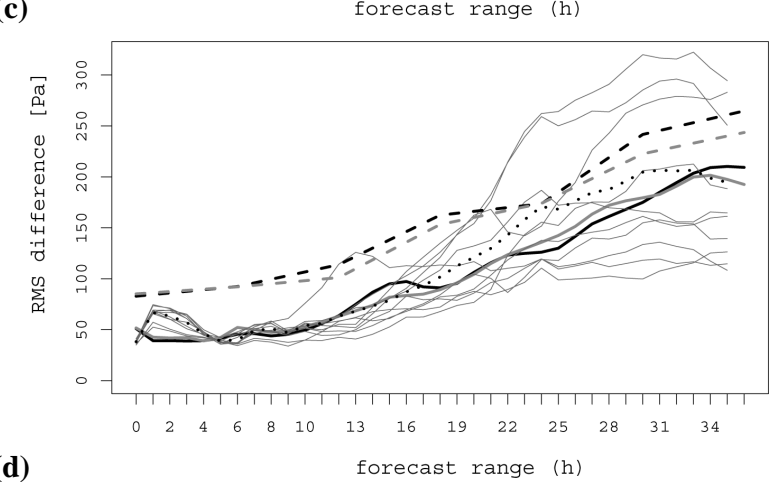

Fig. 4. The same as in Fig. 3 but for mean sea level pressure.

the problem already detected in terms of z500 and ascribed to the evolution of the deep low-pressure system.

Focussing on the 2012102512 case only, the behaviour of the two ensembles is also discussed by analysing the spectra of the perturbations (Fig. 5). Perturbations are computed as the difference between each ensemble member and the ensemble mean in terms of geopotential field at $500 \mathrm{hPa}$. 

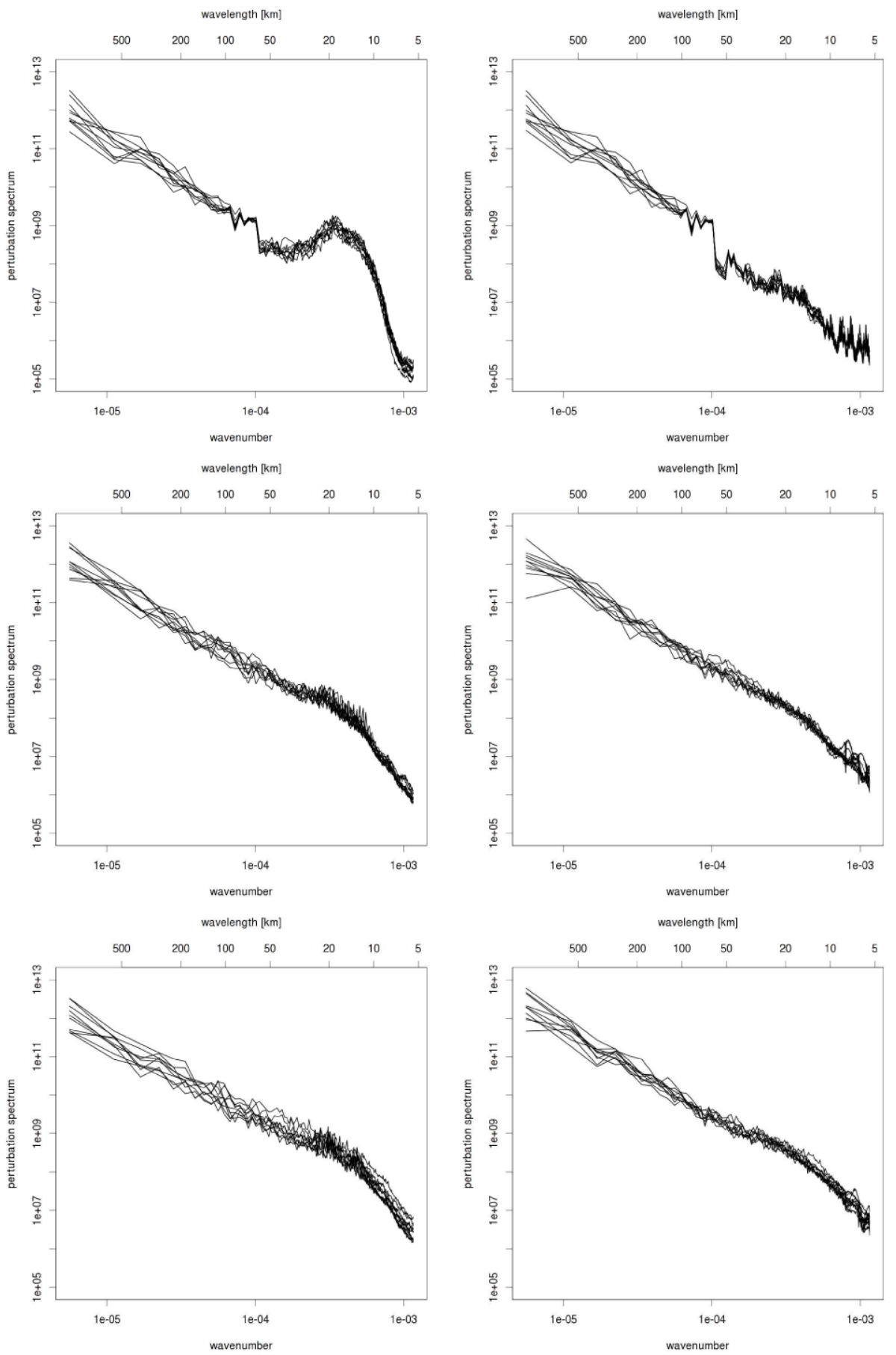

Fig. 5. Perturbation spectra of the field of geopotential at $500 \mathrm{hPa}$ for each member of COSMO-H2-EPS (left panels) and EXP-H2-EPS (right panels) at the initial time (top), and at $+6 \mathrm{~h}$ (middle) and $+12 \mathrm{~h}$ (bottom) forecast range.

Initial state perturbations come, for both ensembles, from the ENS perturbed analyses, to which only the effect of the interpolation is added (Fig. 5, top panels). It is recalled here that the initial conditions of EXP-H2-EPS are directly interpolated from the ECMWF ENS ones, from a 0.25 to 0.025 degree grid, while the initial conditions of COSMO-H2-EPS are interpolated from the COSMO-LEPS initial conditions, which are in turn interpolated from the ECMWF ENS ones, with a two-step interpolation: the former from a 0.25 to 0.0625 degree grid, the latter from the 0.0625 grid to the $0.025 \mathrm{deg}$ grid. The spectra are identical up to a wavenumber around 1e-04, equivalent to a wavelength of about $60 \mathrm{~km}$, approximately equal to the scale resolved by the global ensemble (estimated as double the grid spacing), which provides 
all the larger-scale signals. For smaller scales, the spectra behave quite differently. With the direct interpolation (top right), perturbation amplitude is low on scales smaller than $60 \mathrm{~km}$. The magnitude of the perturbations decreases quite steadily, with some noise especially at the wavelengths between 10 and $5 \mathrm{~km}$, close to the resolution of the $2.8 \mathrm{~km}$ grid. However, with the intermediate step (top left) there is a peak of magnitude in the perturbations at wavelengths between 20 and $10 \mathrm{~km}$, approximately at the scale resolved by the $7 \mathrm{~km}$ model, followed by a sharp decrease and with little signal on the smaller scales. The peak at the $20-10 \mathrm{~km}$ wavelength is the part of the perturbations introduced by the interpolation on the $7 \mathrm{~km}$ grid. After $6 \mathrm{~h}$ of forecast (middle panels), the difference between the two spectra are significantly reduced, both at the $20-10$ and $10-5 \mathrm{~km}$ scale. The two spectra become quite similar after $12 \mathrm{~h}$ (bottom panels), both being characterised by a decrease in the perturbation magnitude for wavelengths below $10 \mathrm{~km}$. Therefore the geopotential perturbations behave similarly from the point of view of how the signal propagates at the different scales. Similar behaviour has been detected for the other cases (not shown).

\subsection{Analysis of the precipitation fields}

The impact of the different nesting approaches on the precipitation forecast is first evaluated by analysing the precipitation patterns, then by performing an objective verification of the ensemble performances against observations.

The difference between 6-hourly precipitation fields forecasted by the members of COSMO-H2-EPS and of EXPH2-EPS has been computed, summing up the RMS differences between pairs of members. This computation has been performed at first on the original grid, then it has been repeated after an upscaling of the fields over boxes which contain an increasing number of grid points: $2 \times 2,4 \times 4, \ldots$, up to $256 \times 256$ points. Finally, the RMS difference has been divided by the spread of the COSMO-H2-EPS ensemble, to provide an indication of its significance with respect to the variability of the reference ensemble. Computation has been performed for each case and for each forecast range separately. In Fig. 6 the ratio between RMS difference and spread is shown for six cases, as a function of the horizontal scale of upscaling, for forecast ranges from $0-6$ up to $30-36 \mathrm{~h}$. It should be noted that the vertical scale is not the same in all the plots. This choice has been made to improve readability and should not affect the analysis of the results, since, as described, RMS difference is normalised by the correspondent spread. In order to identify the scale characterising the difference in the precipitation patterns, the curve decrease is analysed: a marked decrease in the curve (referred to as a "knee" in the curve) indicates which level of upscaling is needed to make the precipitation patterns significantly more similar to each other, compared with the spread of the ensemble. This level of upscaling, expressed in $\mathrm{km}$ of the upscaling box, detects the minimum spatial scale of the differences. When it is not possible to find a "knee", the scale at which the ratio between RMS difference and spread halves is adopted as a quantitative indicator of the scale of the differences. Here only 6 cases are shown, selected in order to represent the full spectrum of different behaviours of the 21 cases.

For the 2012092512 case (Fig. 6a), the differences are quite constant up to a horizontal scale of $8 \times 8$ grid points, then they start to decrease. A more marked decrease takes place for most forecast ranges at scales between 16 and 32 grid points. Therefore, the precipitation patterns forecasted by the two systems have a minimum scale of differences of the order of $24 \mathrm{~km}$, but differences remain relatively high up to $90 \mathrm{~km}$.

For the 2012093012 case (Fig. 6b), different behaviour is detected. The differences decrease quite steadily from the beginning, with a more marked decrease starting from the $4 \times 4$ grid point scale. Therefore, it is difficult to detect a minimum scale for the differences. The scale at which the ratio halves with respect to its original value is around $32 \times 32$ grid points, therefore about $90 \mathrm{~km}$.

For the 201210612 case (Fig. 6c), the curves behave similarly to those of the first case. For all the forecast ranges a sharp decrease is observed, either at $16 \times 16$ or $32 \times 32$ grid point scale. Therefore, the minimum scale of the differences is in the order of 45 or $90 \mathrm{~km}$, depending on the range considered. The $6 \mathrm{~h}$ forecast range should not be considered, since only very light and localised precipitation was forecast and observed.

For the 2012101712 case (Fig. 6d), the behaviour is intermediate: the curves decrease already from the beginning, but the decrease is faster than for the 2012093012 case. The scale at which the ratio halves is between 16 and 32 grid points ( 45 and $90 \mathrm{~km}$ ) for most of the forecast ranges, reducing to about $24 \mathrm{~km}$ only for the $12 \mathrm{~h}$ forecast range.

For the 2012102212 case (Fig. 6e), the curve decrease is initially very slow, then more pronounced. The scale at which the ratio halves is quite large, about 64 points $(180 \mathrm{~km})$ for the forecast ranges 18,24 , and $30 \mathrm{~h}$, while it is smaller for the 12 (about $90 \mathrm{~km}$ ) and 6 (about $45 \mathrm{~km}$ ) h forecast range.

Finally, the 2012103012 case (Fig. 6f) has behaviour similar to that of the 2012101712 case for the first 4 forecast ranges: the curves decrease quite rapidly, especially from the $8 \times 8$ grid point scale, reaching values of half the initial ratio at scales between 45 and $90 \mathrm{~km}$.

In general, two main behaviours are found: for some cases and some forecast ranges it is possible to detect a "knee" in the curve of normalised RMS difference, indicating that a minimum scale exists at which the difference between the precipitation patterns takes place. This scale is of variable size, from about 16 (in one case, not shown) up to about $90 \mathrm{~km}$. For other cases and other forecast ranges, the curve already decreases from the beginning, either quite slowly or more rapidly. In these cases, the scale at which the ratio halves has been considered: values are between 45 and $180 \mathrm{~km}$. Overall, it is found that the difference in the 

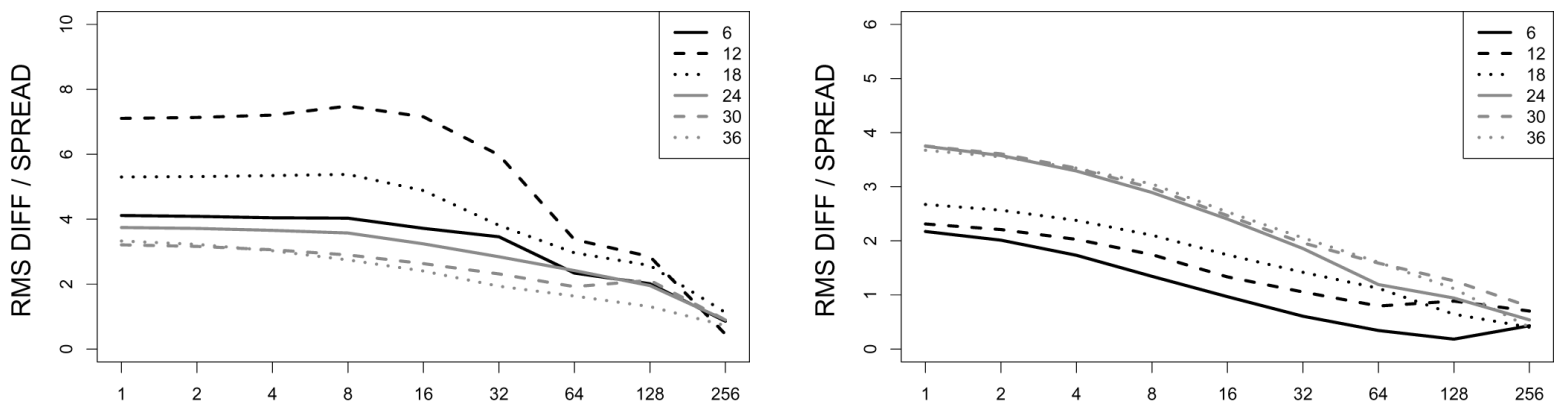

(a)

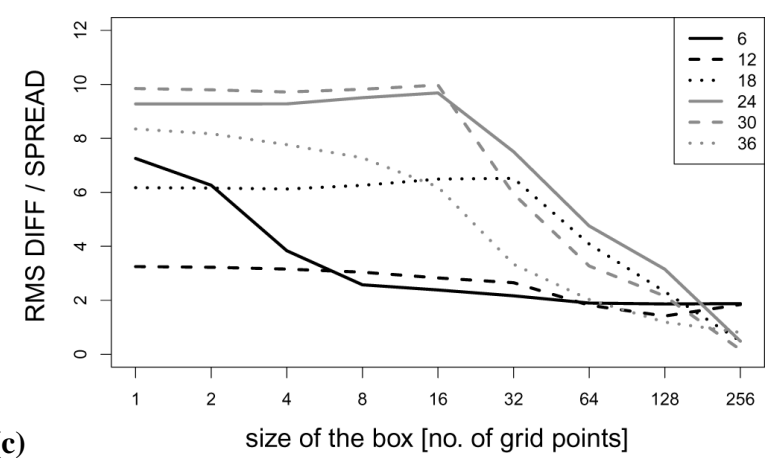

(c)

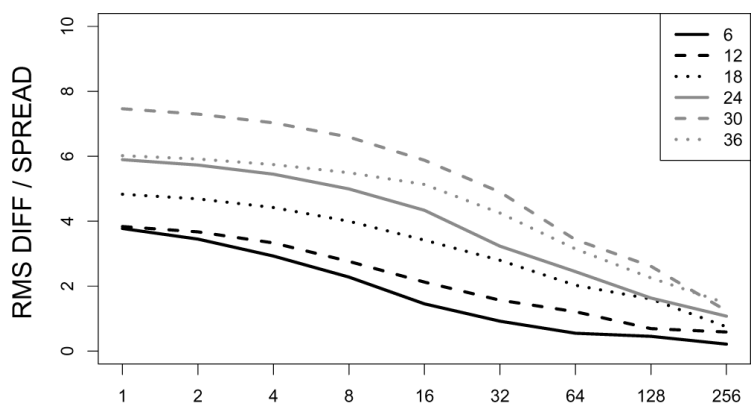

(e) (b)

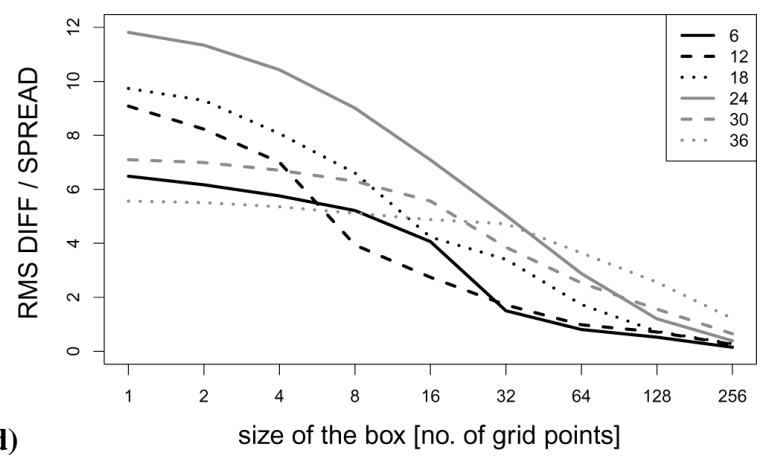

Fig. 6. Ratio between the RMS difference between all the members of the two ensembles and the spread of COSMO-H2-EPS as a function of the horizontal scale of upscaling (indicated as the size, in grid points, of each upscaling box) in terms of $6 \mathrm{~h}$ accumulated precipitation for the different forecast ranges (from 0-6 to 30-36 h). Plots are for the cases (from top left to bottom right): 2012092512, 2012093012, $2012101612,2012101712,2012102212,2012103012$.

precipitation patterns forecasted by pairs of members is not negligible, even if in some cases it might be difficult to detect it properly with a synop observation network. It is not possible to draw conclusions about the influence of the forecast range, since the cases have been examined separately, and precipitation forecast in the different ranges is actually mainly dependent on the evolution of the phenomenon rather than on the forecast range itself.

Since differences are not negligible, an objective verification has also been performed, in order to assess the quality of the precipitation forecasts issued by the two ensembles. The verification period extends from 25 September to 6 November 2012. As already mentioned, the experimental ensemble has only been run for 21 cases in this period, with a $36 \mathrm{~h}$ forecast range. Therefore, the verification sample is slightly smaller than 1 month.

Verification was first performed over the entire domain, in terms of precipitation accumulated over $12 \mathrm{~h}$ compared against synop observations (about 400 stations in the domain), considering the forecast value at the nearest grid point for each observation location.

In Table 1 the values of the ranked probability score (RPS) and skill score (RPSS) are shown for each system and for each forecast range (6-18 and 18-30h). The skill of the two ensembles is comparable, with a little outperformance of EXP-H2-EPS over COSMO-H2-EPS.

In Fig. 7 the Brier skill score (BSS) as a function of the precipitation threshold is shown. The scores are quite close to each other for all thresholds and for both forecast ranges, 
Table 1. Ranked probability score and skill score of the two ensembles computed in terms of $12 \mathrm{~h}$ accumulated precipitation against SYNOP data.

\begin{tabular}{lcccc}
\hline & \multicolumn{2}{c}{$6-18 \mathrm{~h}$} & \multicolumn{2}{c}{$18-30 \mathrm{~h}$} \\
Ensemble system & RPS & RPSS & RPS & RPSS \\
\hline COSMO-H2-EPS & 0.21 & 0.49 & 0.23 & 0.40 \\
EXP-H2-EPS & 0.20 & 0.51 & 0.23 & 0.41 \\
\hline
\end{tabular}

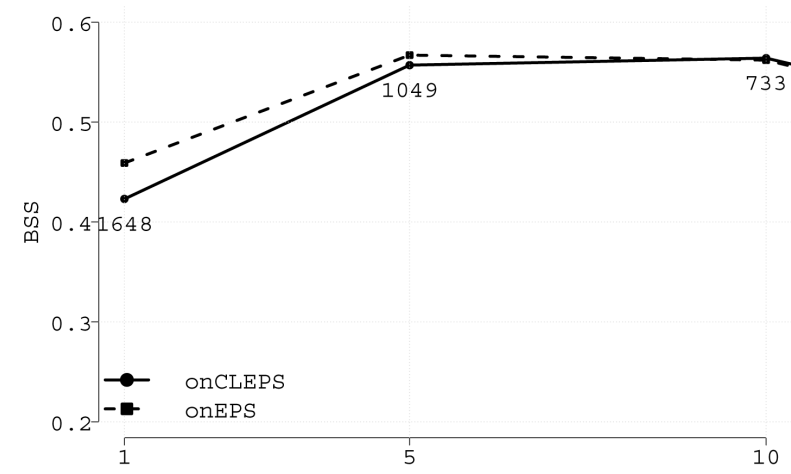

(a)

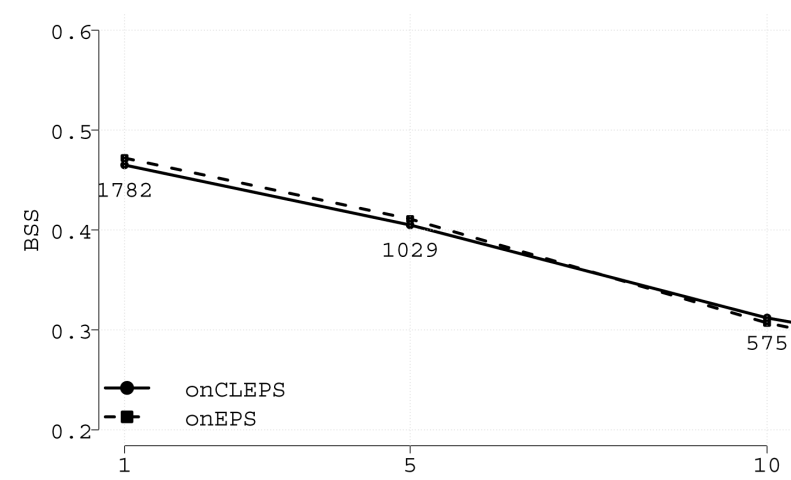

(b)

thresholds (mm/12h)

Fig. 7. Brier skill score of COSMO-H2-EPS (solid line, labelled "onCLEPS") and EXP-H2-EPS (dashed line, labelled "onEPS") as a function of the threshold for the 6-18 h (a) and 18-30 h (b) forecast range. Verification is performed for 12-hourly precipitation. Numbers indicate the observed occurrences of each event.

EXP-H2-EPS outperforming COSMO-H2-EPS for light precipitation at the first forecast range $(6-18 \mathrm{~h})$. The number of events for each threshold is also reported in the plots.

Since some heavy precipitation events took place over northern and central Italy during the analysed period, a different verification has also been performed, aimed at evaluating the impact of the LBC diversity focussing on intense precipitation cases. Precipitation accumulated over $6 \mathrm{~h}$ has been considered and scores have been computed against observations collected by a dense network covering northern and central Italy (about 850 stations). In order to perform a verification more suitable for high-resolution forecast of intense events, a different methodology has been adopted, con-

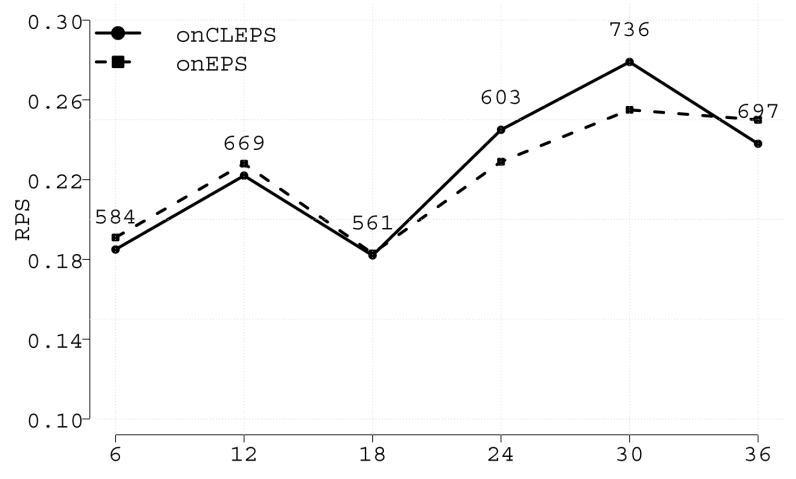

(a)

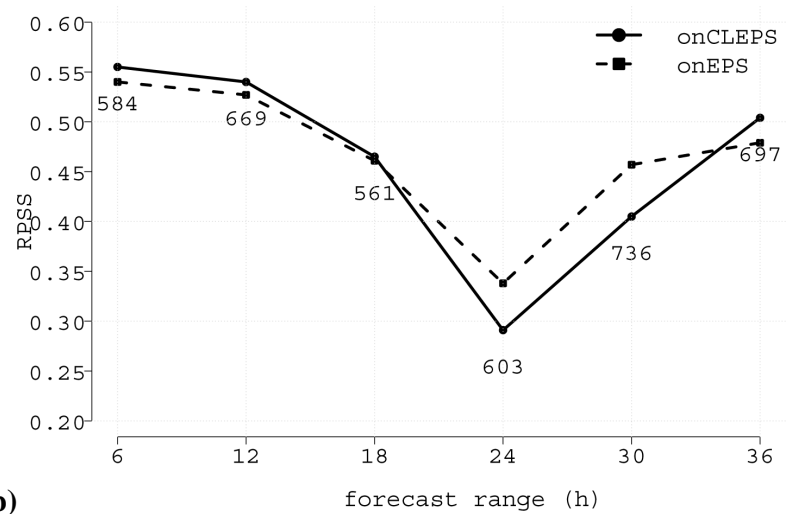

Fig. 8. Ranked probability score (a) and skill score (b) of COSMO-H2-EPS (solid line, labelled "onCLEPS") and EXP-H2EPS (dashed line, labelled "onEPS") as a function of the forecast range. Verification is performed for 6-hourly precipitation over northern and central Italy.

sisting in an upscaling of both forecast and observed values (Marsigli et al., 2008). The verification area has been covered with boxes of 0.2 degrees $\times 0.2$ degrees, then the averages (one for each member) of the values forecasted on the grid points falling in each box are compared with the average of the values observed on the stations falling in the same box, provided that a box contains at least 3 stations.

Both RPS and RPSS indicate that the skill of the two ensembles is comparable (Fig. 8), EXP-H2-EPS slightly outperforming COSMO-H2-EPS at the 24 and $30 \mathrm{~h}$ forecast ranges. This dependence on the forecast range is ascribed to the performance of the two systems over a few intense events, which took place at these ranges, rather than on the forecast range itself. In order to check the dependency on the precipitation intensity, the Brier skill score is also shown, for the 1, 5, and $10 \mathrm{~mm}$ thresholds (Fig. 9).

While for the $1 \mathrm{~mm}$ threshold the scores are quite comparable (Fig. 9a), for the higher thresholds EXP-H2-EPS has higher BSS than COSMO-H2-EPS at the 24 and $30 \mathrm{~h}$ forecast ranges, confirming that the outperformance is mainly due to a better forecast of high precipitation. 


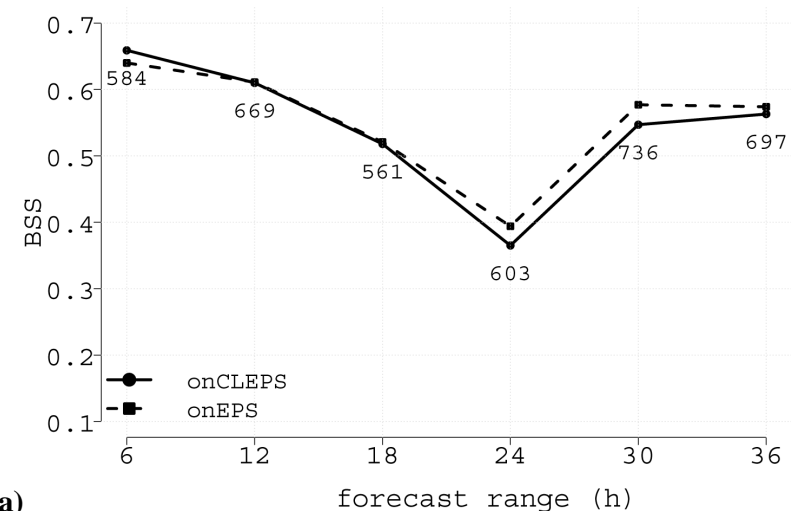

(a)

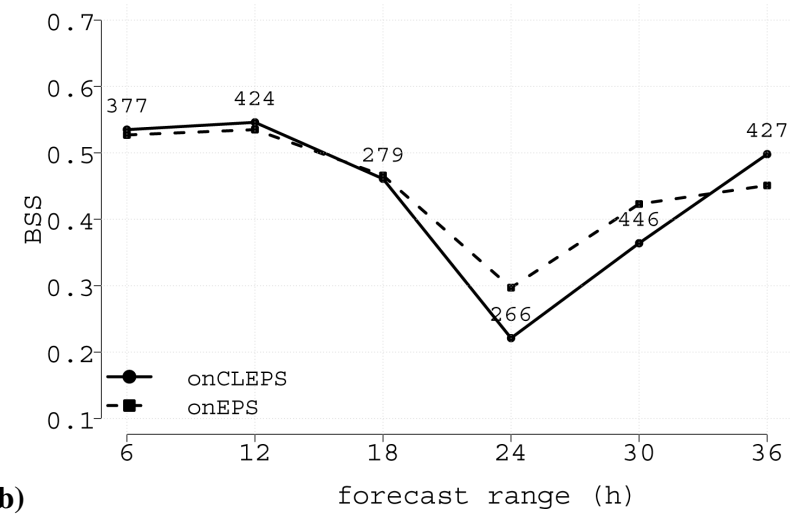

(b)

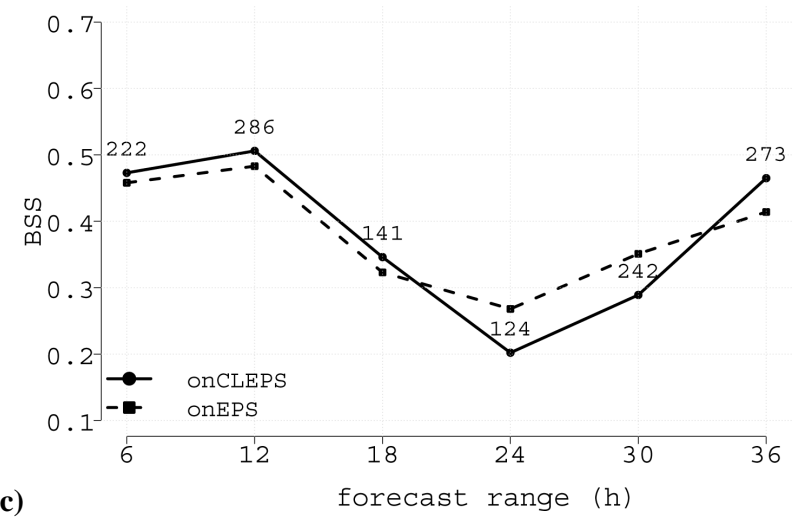

Fig. 9. The same as in Fig. 8 but for the Brier skill score. Thresholds are: $1 \mathrm{~mm}(\mathbf{a}), 5 \mathrm{~mm}$ (b), and $10 \mathrm{~mm}$ (c).

\section{Summary and conclusions}

An analysis has been undertaken of the performance of two different nesting approaches for convection-permitting ensemble forecasting. The reference approach, adopted in the COSMO-H2-EPS ensemble, consists of providing ICs and LBCs to the $2.8 \mathrm{~km}$ ensemble by a coarser-resolution ensemble based on the same model. The experimental approach, implemented in the EXP-H2-EPS ensemble, is a direct nesting, where ICs and LBCs are provided by a global ensemble, run at quite coarser resolution. The purpose of this work was to investigate the effect of the direct nesting approach, compared with the one based on the intermediate step, for a specific application, which is ensemble forecasting over Italy with the main focus on the forecast of intense precipitation. Analysis has been performed in terms of geopotential at $500 \mathrm{hPa}$ and precipitation.

Results show that the variability introduced in the geopotential fields by the direct nesting is often smaller than the uncertainty described by the reference ensemble, represented by the ensemble spread. When the forecast error is considered, expressed by the RMSE of the ensemble mean, it appears that the differences between pairs of members having different nesting approaches are generally smaller than this error, therefore hardly detectable from ensemble forecasting evaluation. A case study-based evaluation highlights that few cases show a different behaviour. In four cases the experimental nesting approach leads to a better performance of the ensemble, due to the better performance of one member. Nevertheless, in two other cases, an additional error is introduced on two ensemble members with the direct nesting. These results are confirmed by a similar analysis carried out in terms of mean sea level pressure.

Considering the relation between spread and error over the whole sample, it is found that the direct nesting leads to an improved relation, with spread slightly increased and error significantly reduced.

An analysis of the perturbation spectra indicates that the geopotential perturbations behave similarly in the two approaches, from the point of view of the way the signal propagates at the different scales. Both ensembles are characterised by little signal on the small scales at initial time, as expected since ICs are downscaled from analyses perturbed at coarse resolution. Nevertheless, this feature will not affect the $2.8 \mathrm{~km}$ ensemble in its operational set-up, since highresolution perturbed analyses derived from KENDA will be used as ICs.

In terms of precipitation, it is found that forecasts issued by members with different nesting approaches generally differ on spatial scales which are highly dependent on the case, ranging from 16 up to $180 \mathrm{~km}$. Though these differences are not negligible, it might be difficult to evaluate their impact on the forecast quality, since spatial errors in the precipitation fields may reach scales of $50-100 \mathrm{~km}$ even if forecasted by a high-resolution model.

The skill of the ensemble precipitation forecasts has been evaluated over the whole domain against synop data, showing comparable performances of the two ensembles. Highresolution verification has also been performed over a restricted domain, where few intense events took place and a dense observation network was available. Results show that the skill of the two ensembles is similar, with a little outperformance of the direct-nesting ensemble for intense precipitation. This evaluation points out that the overall quality of the $2.8 \mathrm{~km}$ ensemble in terms of precipitation forecast is not badly affected by the provision of lower resolution LBCs from the global ensemble. 
This work should be regarded as the first step of a deeper analysis, where more cases will be considered, especially including different weather situations. Furthermore, it is intended to study the impact of spatial and temporal resolution separately, considering the usefulness of hourly boundary conditions provided by the global ensemble.

Acknowledgements. This work was partly supported by the Italian Civil Protection Department, in the framework of the MODMDET3 Project. The Italian institutions providing observed precipitation data are also acknowledged. The authors are grateful to Jun $\mathrm{Du}$ and to two anonymous referees for their valuable comments, which allowed them to improve the manuscript greatly.

Edited by: R. Buizza

Reviewed by: J. Du and two anonymous referees

\section{References}

Amengual, A., Romero, R., Homar, V., Ramis, C., and Alonso, S.: Impact of the lateral boundary conditions resolution on dynamical downscaling of precipitation in mediterranean Spain, Clim. Dynam., 29, 487-499, doi:10.1007/s00382-007-0242-0, 2007.

Buizza, R., Houtekamer, P. L.,Toth, Z.,Pellerin, G., Wei, M., and Zhu, Y.: A Comparison of the ECMWF, MSC, and NCEP Global Ensemble Prediction Systems, Mon. Weather Rev., 133, 10761097, 2005.

Davies, T.: Lateral boundary conditions for limited-area models, Q. J. Roy. Meteor. Soc., 140, 185-196, doi:10.1002/qj.2127, 2014.

de Elia, R., Laprise, R., and Denis, B.: Forecasting skill limits of nested, limited-area models: a perfect-model approach, Mon. Weather Rev., 130, 2006-2023, 2002.

Denis, B., Laprise, R., Côté, J., and Caya, D.: Downscaling ability of one-way nested regional climate models: The big-brother experiment, Clim. Dynam., 18, 627-646, 2001.

Ducrocq, V., Braud, I., Davolio, S., Ferretti, R., Flamant, C., Jansa, A., Kalthoff, N., Richard, E., Taupier-Letage, I., Ayral, P.-A., Belamari, S., Berne, A., Borga, M., Boudevillain, B., Bock, O., Boichard, J.-L., Bouin, M.-N., Bousquet, O., Bouvier, C., Chiggiato, J., Cimini, D., Corsmeier, U., Coppola, L., Cocquerez, P., Defer, E., Delanoë, J., Di Girolamo, P., Doerenbecher, A., Drobinski, P., Dufournet, Y., Fourrié, N., Gourley, J. J., Labatut, L., Lambert, D., Le Coz, J., Marzano, F. S., Molinié, G., Montani, A., Nord, G., Nuret, M., Ramage, K., Rison, B., Roussot, O., Said, F., Schwarzenboeck, A., Testor, P., Van Baelen, J., Vincendon, B., Aran, M., and Tamayo, J.: HyMeX-SOP1, the field campaign dedicated to heavy precipitation and flash flooding in the northwestern Mediterranean, B. Am. Meteorol. Soc., online first, doi:10.1175/BAMS-D-12-00244.1, 2013

Ferretti, R., Pichelli, E., Gentile, S., Maiello, I., Cimini, D., Davolio, S., Miglietta, M. M., Panegrossi, G., Baldini, L., Pasi, F., Marzano, F. S., Zinzi, A., Mariani, S., Casaioli, M., Bartolini, G., Loglisci, N., Montani, A., Marsigli, C., Manzato, A., Pucillo, A., Ferrario, M. E., Colaiuda, V., and Rotunno, R.: Overview of the first HyMeX Special Observation Period over Italy: observations and model results, Hydrol. Earth Syst. Sci. Discuss., 10, 11643-11710, doi:10.5194/hessd-10-11643-2013, 2013.
Gerard, L., Piriou, J.-M., Brožková, R., Geleyn, J.-F., and Banciu D.: Cloud and Precipitation Parameterization in a Meso-GammaScale Operational Weather Prediction Model, Mon. Weather Rev., 137, 3960-3977, 2009.

HYMEX - HYdrological cycle in the Mediterranean EXperiment, Hymex Project, available at: www.hymex.org, last access: 4 March 2014, 2010-2020.

Marsigli, C., Montani, A., Nerozzi, F., Paccagnella, T., Tibaldi, S., Molteni, F., and Buizza, R.: A strategy for High-Resolution Ensemble Prediction. Part II: Limited-area experiments in four Alpine flood events, Q. J. Roy. Meteor. Soc., 127, 2095-2115, 2001.

Marsigli, C., Montani, A., and Paccagnella, T.: A spatial verification method applied to the evaluation of high-resolution ensemble forecasts, Meteorol. Appl., 15, 125-143, 2008.

Marsigli, C., Montani, A., and Paccagnella, T., 2013. Test of a COSMO-based convection-permitting ensemble in the Hymex framework, COSMO Newsletter No. 13, available at: http://www.cosmo-model.org/content/model/documentation/ newsLetters/default.htm, last access: 4 March 2014, 2013

Molteni, F., Buizza, R., Marsigli, C., Montani, A., Nerozzi, F., and Paccagnella, T.: A strategy for High-Resolution Ensemble Prediction. Part I: Definition of Representative Members and Global Model Experiments, Q. J. Roy. Meteor. Soc., 127, 2069-2094, 2001.

Montani, A., Cesari, D., Marsigli, C., and Paccagnella, T.: Seven years of activity in the field of mesoscale ensemble forecasting by the COSMO-LEPS system: main achievements and open challenges, Tellus A, 63, 605-624, 2011.

Nutter, P., Stensrud, D., and Xue, M.: Effects of coarsely resolved and temporally interpolated lateral boundary conditions on the dispersion of limited-area ensemble forecasts, Mon. Weather Rev., 132, 2358-2377, 2004.

Reich, H., Rhodin, A., and Schraff, C.: LETKF for the nonhydrostatic regional model COSMO-DE, COSMO Newsletter No. 11, available at: http://www.cosmo-model.org/content/model/ documentation/newsLetters/default.htm, last access: 4 March 2014, 2011.

Steppeler, J., Doms, G., Schattler, U., Bitzer, H. W., Gassmann, A., Damrath, U., and Gregoric, G.: Meso-gamma scale forecasts using the nonhydrostatic model LM, Meteorol. Atmos. Phys., 82, 75-96, 2003.

Termonia, P.: Monitoring and improving the temporal interpolation of lateral-boundary coupling data for limited-area models, Mon. Weather Rev., 131, 2450-2463, 2003.

Termonia, P., Deckmyn, A., and Hamdi, R.: Study of the lateral boundary condition temporal resolution problem and a proposed solution by means of boundary error restarts, Mon. Weather Rev., 137, 3551-3566, 2009.

Warner, T. T., Peterson, R. A., and Treadon, R. E.: A tutorial on lateral boundary conditions as a basic and potentially serious limitation to regional numerical weather prediction, B. Am. Meteorol. Soc., 78, 2599-2617, 1997. 